\title{
Sphingosine 1-phosphate release from platelets during clot formation: close correlation between platelet count and serum sphingosine 1-phosphate concentration
}

\author{
Yoshikazu Ono ${ }^{1,3}$, Makoto Kurano ${ }^{2}$, Ryunosuke Ohkawa ${ }^{1}$, Hiromitsu Yokota ${ }^{1}$, Koji Igarashi ${ }^{4}$, Junken Aoki ${ }^{5}$,
} Minoru Tozuka ${ }^{3}$ and Yutaka Yatomi ${ }^{1,2^{*}}$

\begin{abstract}
Background: Sphingosine 1-phosphate (Sph-1-P), abundantly stored in platelets and released extracellularly upon activation, plays important roles as an extracellular mediator by interacting with specific cell surface receptors, especially in the area of vascular biology and immunology/hematology. Although the plasma Sph-1-P level is reportedly determined by red blood cells (RBCs), but not platelets, this may not be true in cases where the platelets have been substantially activated.

Methods and results: We measured the Sph-1-P and dihydrosphingosine 1-phosphate (DHSph-1-P) levels in serum samples (in which the platelets had been fully activated) from subjects with $(n=21)$ and without $(n=33)$ hematological disorders. We found that patients with essential thrombocythemia exhibited higher serum Sph-1-P and DHSph-1-P concentrations. The serum Sph-1-P concentration was closely correlated with the platelet count but was very weakly correlated with the RBC count. Similar results were obtained for DHSph-1-P. The serum Sph-1-P and DHSph-1-P levels were inversely correlated with the level of autotaxin (ATX), a lysophosphatidic acid-producing enzyme. A multiple regression analysis also revealed that the platelet count had the greatest explanatory impact on the serum Sph-1-P level.

Conclusions: Our present results showed close correlations between both the serum Sph-1-P and DHSph-1-P levels and the platelet count (but not the RBC count); these results suggest that high concentrations of these sphingoid base phosphates may be released from platelets and may mediate cross talk between platelet activation and the formation of atherosclerotic lesions.
\end{abstract}

Keywords: Sphingosine 1-phosphate, Dihydrosphingosine 1-phosphate, Platelets, Red blood cells, Autotaxin

\section{Introduction}

Sphingosine 1-phosphate (Sph-1-P) has been shown to be an important lipid mediator in the fields of vascular biology and immunology/hematology [1]. Although Sph1-P has been demonstrated to act as an intracellular mediator, most of the physiological functions of Sph-1-P are exerted through S1P receptors located on the plasma

\footnotetext{
* Correspondence: yatoyuta-tky@umin.ac.jp

${ }^{1}$ Department of Clinical Laboratory, The University of Tokyo Hospital, Tokyo, Japan

2Department of Clinical Laboratory Medicine, Graduate School of Medicine, The University of Tokyo, 7-3-1 Hongo, Bunkyo-ku, Tokyo 113-8655, Japan Full list of author information is available at the end of the article
}

membrane $[2,3]$. Therefore, elucidating the mechanism that regulates plasma Sph-1-P levels is an important task. Regarding the source of Sph-1-P in plasma, a previous report proposed that the plasma circulating Sph-1-P level might be determined by platelets, since Sph-1-P is abundantly accumulated in platelets [4]. However, from experiments using genetically modified mice models [5] and analyses in human subjects [6], the plasma Sph-1-P level is now thought to be determined not by platelets, but mainly by erythrocytes. This theory seems plausible because the circulating volume of erythrocytes is much greater than that of platelets. 
Although erythrocytes determine the plasma Sph-1-P levels during the steady state in healthy subjects, however, platelets may also play an important role during pathological states in which the platelets are activated, such as in thrombosis. Regarding this possibility, the clinical significance of Sph-1-P in atherosclerotic diseases differs depending on the type of samples in which the Sph-1-P level is measured: plasma HDL-linked Sph-1-P, albumin-linked Sph-1-P, or serum Sph-1-P. Clinical studies investigating Sph-1-P levels in atherosclerotic diseases have reported that although HDL linked Sph-1-P is negatively related with atherosclerosis [7,8], Sph-1-P in non-HDL fractions and serum Sph-1-P are positively correlated with atherosclerosis $[7,9]$. This discrepancy might be explained by the different origin of Sph-1-P in these samples; contrary to plasma samples in which the Sph-1-P levels are mainly determined by erythrocyte-derived Sph-1-P, platelets can have a large impact on the Sph-1-P concentration in serum samples in which the platelets are fully activated. If true and considering that Sph-1-P released from platelets might be preferably carried on albumin (non-HDL fraction) [10,11], these results suggest that platelet-derived Sph-1-P might exert proatherosclerotic properties. To our knowledge, the factors that determine the serum Sph-1-P levels have not yet been elucidated. In the present study examining serum samples from subjects with and without hematological disorders, we investigated the factors that determine the serum Sph-1-P levels. We also examined dihydrosphingosine 1-phosphate (DH-Sph-1-P), which is considered to work as an agonist against $\mathrm{Sph}-1-\mathrm{P}$ receptors [12].

\section{Results}

Serum Sph-1-P and DHSph-1-P levels were increased in patients with essential thrombocythemia (ET)

To elucidate which factors determine the serum Sph-1-P and DHSph-1-P levels, we first measured the serum Sph-1-P and DHSph-1-P levels in subjects with hematological disorders and with non-hematological disorders (NHD) (Figure 1). The basic characteristics of the subjects are shown in Table 1. As predicted by the fact that erythrocytes determine the plasma Sph-1-P levels to a large degree [6], the serum Sph-1-P levels were lower in the aplastic anemia (AA) group, which had a significantly lower RBC count, compared with the other groups. Importantly, the ET group had a significantly higher Sph-1-P and DHSph-1-P level, although the RBC count was similar to those of the other groups except for the AA group. The platelet count for the ET group was much higher than those in the other groups (Table 1). Therefore, these results suggested that platelet count might also determine the serum Sph-1-P and DHSph-1-P levels.

\section{Serum Sph-1-P and DHSph-1-P concentrations were more positively related with the platelet count than with the RBC count}

Next, we compared the serum Sph-1-P and DHSph-1-P levels with the $\mathrm{RBC}$ count or the platelet count. As shown in Figure 2B, the serum concentration of Sph-1-P was significantly correlated with the $\mathrm{RBC}$ count $(\mathrm{r}=$ 0.393, $P=0.003)$. However, unlike the plasma Sph-1-P level, the serum Sph-1-P level was more significantly correlated with the platelet count $(r=0.775, P<0.001$,

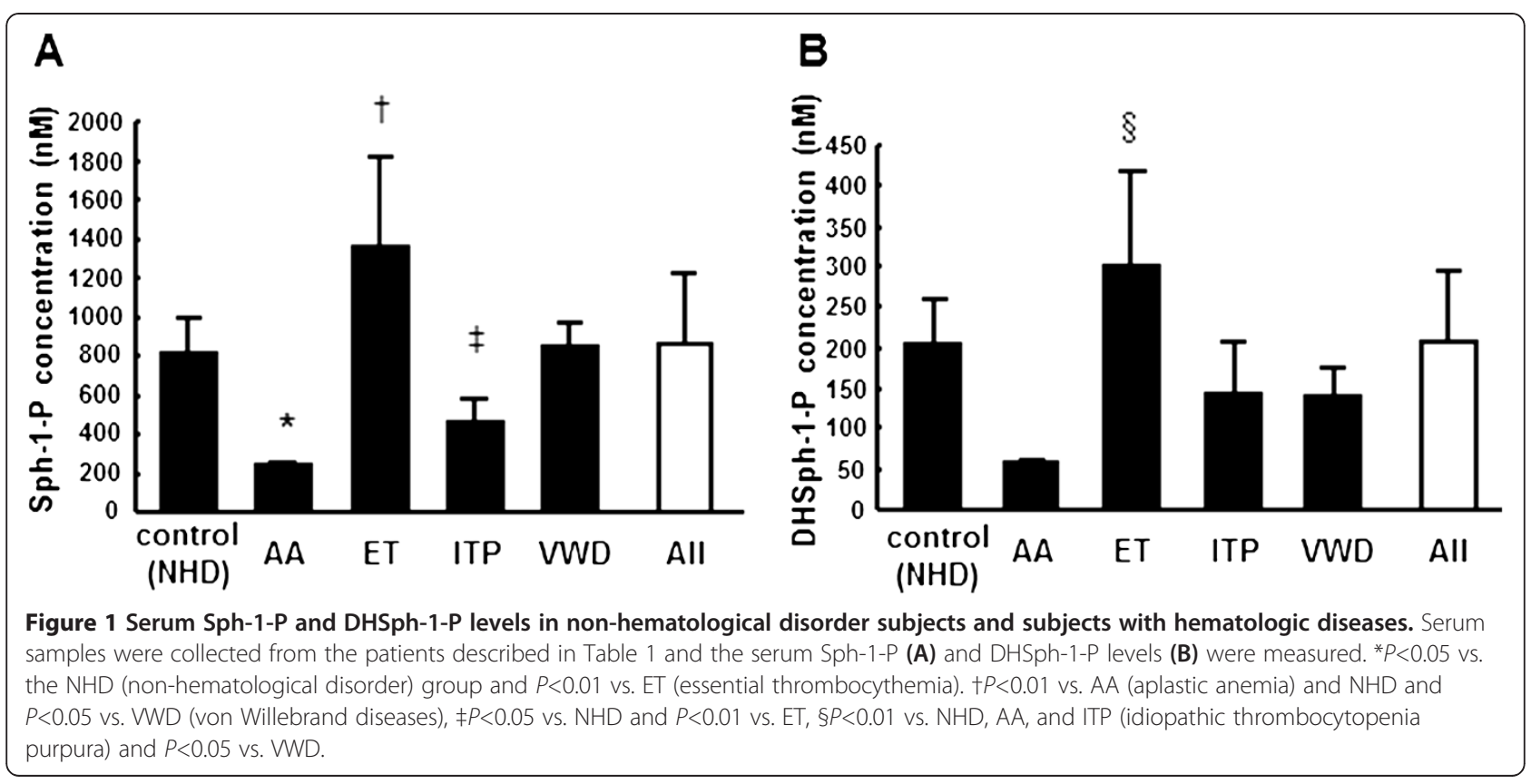


Table 1 Basic characteristics of the subjects

\begin{tabular}{lccccccc}
\hline & RBC $(\mathbf{X} \mathbf{1 0} / \boldsymbol{\mu L})$ & Platelets $(\mathbf{X} \mathbf{1 0} / \boldsymbol{\mu L})$ & alb $(\mathbf{m g} / \mathbf{d L})$ & HDL-C $(\mathbf{m g} / \mathbf{d L})$ & LDL-C $(\mathbf{m g} / \mathbf{d L})$ & TG $(\mathbf{m g} / \mathbf{d L})$ & $\mathbf{A T X}(\mathbf{n g} / \mathbf{m L})$ \\
\hline AA & $215.0 \pm 9.9^{*}$ & $2.8 \pm 0.5$ & $4.4 \pm 0.1$ & $54.8 \pm 14.6$ & $103.6 \pm 35.4$ & $178.5 \pm 200.1$ & $1.49 \pm 0.39$ \\
\hline ET & $467.9 \pm 101.2$ & $84.8 \pm 35.4 \dagger$ & $4.2 \pm 0.2$ & $56.8 \pm 14.4$ & $104.5 \pm 40.4$ & $144.7 \pm 66.5$ & $0.96 \pm 0.13$ \\
\hline ITP & $472.2 \pm 40.1$ & $3.1 \pm 1.1$ & $4.1 \pm 0.4$ & $57.9 \pm 15.7$ & $100.9 \pm 25.9$ & $164.2 \pm 127.3$ & $0.96 \pm 0.13$ \\
\hline WWD & $423.0 \pm 89.9$ & $27.5 \pm 6.3$ & $4.4 \pm 0.2$ & $28.9 \pm 0.4$ & $73.9 \pm 59.9$ & $127.7 \pm 29.6$ & $0.95 \pm 0.12$ \\
\hline NHD & $436.4 \pm 71.8$ & $22.1 \pm 8.2$ & $4.1 \pm 0.5$ & $40.3 \pm 19.0$ & $127.4 \pm 53.5$ & $135.7 \pm 71.7$ & $0.90 \pm 0.07$ \\
\hline All & $437.3 \pm 86.6$ & $31.2 \pm 31.1$ & $4.1 \pm 0.4$ & $45.1 \pm 18.9$ & $116.0 \pm 49.6$ & $140.3 \pm 78.7$ & $0.95 \pm 0.05$ \\
\hline
\end{tabular}

Serum samples were collected from subjects with NHD (non-hematological disorders, $n=33$ ) and patients with AA (aplastic anemia, $n=2$ ), ET

(essential thrombocythemia, $n=10$ ), ITP (idiopathic thrombocytopenia purpura, $n=6$ ), and VWD (von Willebrand diseases, $n=3$ ). ATX represents autotaxin. Data were shown as the mean \pm SD.

${ }^{*} P<0.01$ vs. ET, ITP, NHD, and $P<0.05$ vs. VWD; $+P<0.01$ vs. the other groups.

Figure 2A) than with the RBC count. Similar results were observed for the serum DHSph-1-P level (Figure 2C and D). These results suggested that the serum Sph-1-P and the DHSph-1-P levels might derive largely from activated platelets in prepared serum samples.

We also evaluated the correlation between the serum Sph-1-P level and the platelet count in NHD subjects or subjects with hematological disorders separately. In samples from NHD subjects alone, a close correlation between the Sph-1-P or DHSph-1-P level and the platelet count was observed $(r=0.668, P<0.001$, Figure $3 \mathrm{~A} ; \mathrm{r}=$ $0.587, P<0.001$, respectively). Also in samples from subjects with hematological disorders, a significant close correlation between the Sph-1-P level and the platelet
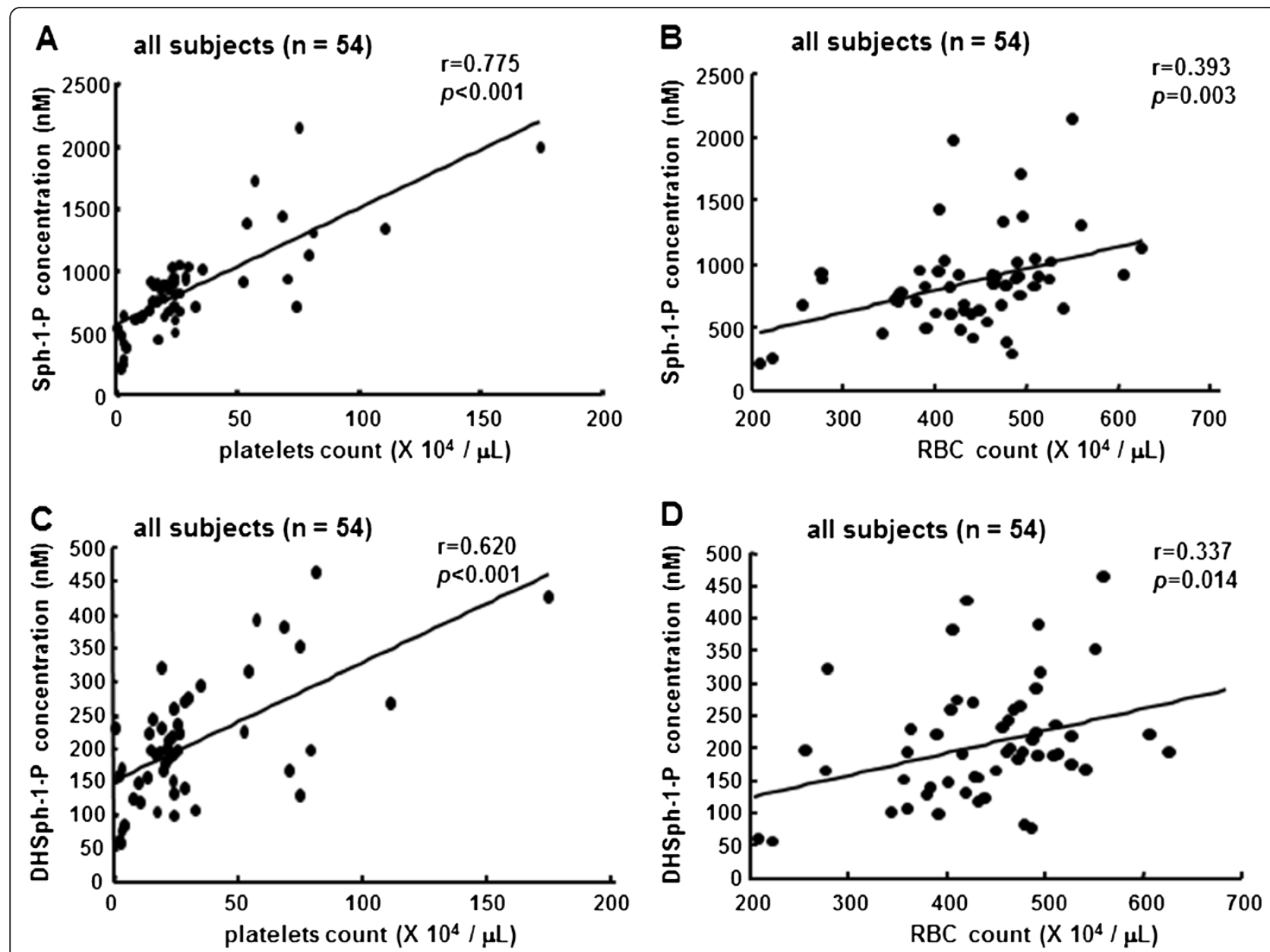

Figure 2 Correlations between serum Sph-1-P level or serum DHSph-1-P level and platelet count or RBC count in all subjects. The correlations between the serum Sph-1-P level and the platelet count ( $\mathbf{A})$ and the RBC count (B) and between the serum DHSph-1-P level and the platelet count $(\mathbf{C})$ and the RBC count $(\mathbf{D})$ in all subjects $(n=54)$ are shown. 


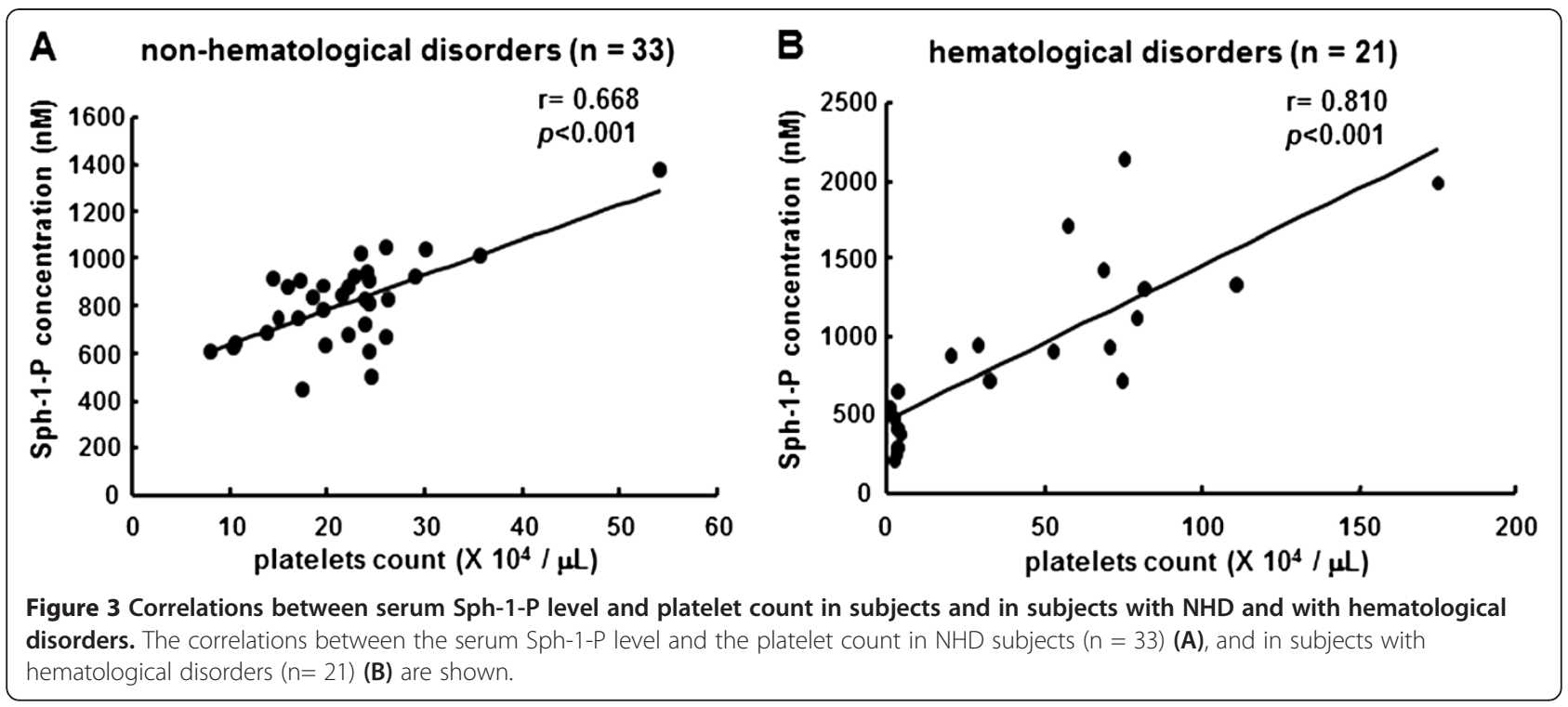

count was observed ( $\mathrm{r}=0.810, P<0.001$, Figure 3B). These results suggest that the serum Sph-1-P and DHSph-1-P levels are mainly determined by the platelet count irrespective of the presence of hematological disorders.

Serum Sph-1-P and DHSph-1-P concentrations were inversely related with the serum ATX level

We further investigated possible determinants of the Sph1-P and DHSph-1-P concentrations. First, we investigated the correlation of Sph-1-P or DHSph-1-P with the HDL cholesterol and albumin levels, since Sph-1-P is mostly bound to HDL or albumin in plasma [13,14]. However, no significant correlations were observed with HDL cholesterol and albumin levels (data not shown).

We next evaluated the correlation between Sph-1-P or DHSph-1-P and the autotaxin (ATX) level. ATX is a key enzyme in the production of lysophosphatidic acid (LPA) from lysophosphatidylcholine (LPC) [15,16]. Along with LPA synthesis, Sph-1-P and DHSph-1-P can theoretically be formed with ATX from sphingosylphosphorylcholine (SPC) [17]. However, contrary to our expectations, the serum ATX level was significantly and inversely related to the serum Sph-1-P and DHSph-1-P concentrations ( $\mathrm{r}=-0$ .407, $P=0.010$ and $\mathrm{r}=-0.452, P<0.001$, respectively) (Figure $4 \mathrm{~A}$ and $\mathrm{B}$ ).
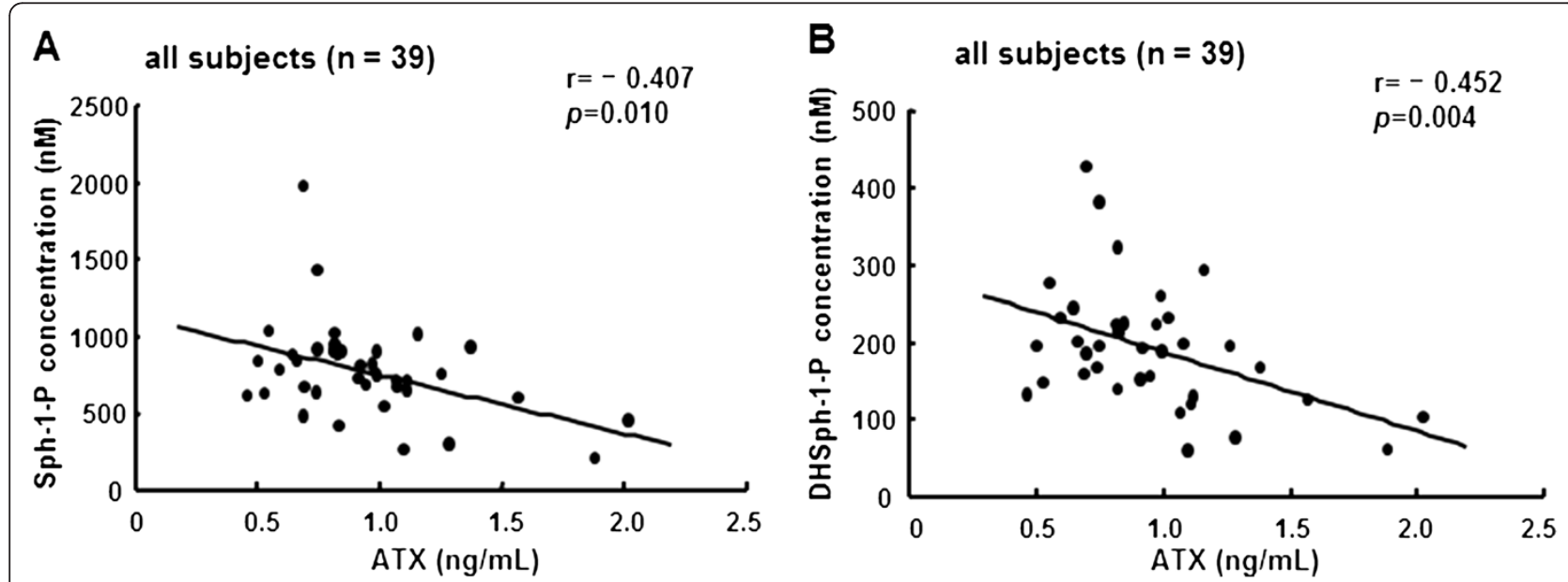

Figure 4 Correlations between the serum Sph-1-P level or the DHSph-1-P level and the ATX level. The correlations between the serum Sph-1-P level (A) or the DHSph-1-P (B) level and the ATX level in all subjects $(n=39)$ are shown. 


\section{Multiple regression analysis revealed that the platelet count was the most important explanatory variable determining the serum Sph-1-P and DHSph-1-P concentrations}

Finally, we performed a multiple regression analysis utilizing the serum Sph-1-P or DHSph-1-P level as the objective variable, as mentioned in the Materials and methods section. As predicted from the correlation studies, the platelet count was the most important explanatory variable for the Sph-1-P concentration, followed by the ATX level and RBC count in this order (Table 2). A similar result was obtained for DHSph-1-P, except for the RBC count (Table 3).

\section{Discussion}

Sphingosine 1-phosphate (Sph-1-P) plays important roles as an extracellular mediator through its interactions with the G protein-coupled receptor S1P1-5, which is expressed on the cell surface; these actions are especially pertinent in the fields of vascular biology, immunology, and hematology. Although erythrocytes are the major determinant of the plasma Sph-1-P level $[5,6]$, the major determinant of the serum Sph-1-P concentration has not yet been elucidated. In the present study, we attempted to elucidate the source of serum Sph-1-P. We also investigated the determinants of the DHSph-1-P concentration, which are thought to act similarly as a ligand for Sph-1-P receptors as Sph-1-P [12].

In this study, we demonstrated that the serum Sph-1-P level was closely related to the platelet count (Figure 2). This finding seems to be reasonable because when the results were adjusted according to the phospholipid level, the platelets contained a higher concentration of Sph-1-P than the erythrocytes [4] and the platelets were fully activated in the serum samples. Multiple regression studies also revealed that the platelet count was the most explanatory variable for Sph-1-P (Table 2).

To our knowledge, this report is the first to demonstrate that the serum Sph-1-P level is determined by the platelet count. This result provides important clues related to the physiological properties of Sph-1-P in

Table 2 Multiple regression analysis for serum Sph-1-P level in all the subjects

\begin{tabular}{lccc}
\hline & b 95\% Cl & Standardized $\boldsymbol{\beta}$ & $\boldsymbol{P}$ value \\
\hline Platelets & $8.17(6.44-9.91)$ & 0.791 & $<0.001$ \\
\hline ATX & $-196.40(-361.46--31.35)$ & -0.219 & 0.021 \\
\hline RBC & $0.78(0.10-1.47)$ & 0.208 & 0.027 \\
\hline HDL-C & & 0.196 \\
\hline TG & & 0.312 \\
\hline LDL-C & & 0.721 \\
\hline Albumin & 0.857 \\
\hline
\end{tabular}

Table 3 Multiple regression analysis for serum DHSph-1-P level in all the subjects

\begin{tabular}{lccc}
\hline & b 95\% Cl & Standardized $\boldsymbol{\beta}$ & $\boldsymbol{P}$ value \\
\hline Platelets & $1.41(0.73-2.09)$ & 0.532 & $<0.001$ \\
\hline ATX & $-86.20(-145.04--27.37)$ & -0.378 & 0.021 \\
\hline RBC & & 0.253 \\
\hline HDL-C & & 0.703 \\
\hline TG & & 0.223 \\
\hline LDL-C & & 0.604 \\
\hline Albumin & & 0.604 \\
\hline
\end{tabular}

atherosclerotic diseases. In the physiological state, cells involved in atherogenesis, such as endothelial cells, macrophages, and smooth muscle cells, might be exposed to Sph-1-P concentrations corresponding to the plasma Sph-1-P level (300-500 nM). However, as reported in this study, the Sph-1-P concentration in serum samples in which the platelets are fully activated was much higher (around 800 to $1,000 \mathrm{nM}$ ) than the plasma Sph-1 -P level. Therefore, cells are likely exposed to higher concentrations of Sph-1-P at the exact site where thrombosis is occurring. This difference in the Sph-1-P concentrations between plasma and the exact site of thrombosis may explain the apparently contradictory clinical influences of serum Sph-1-P and plasma Sph-1-P on atherosclerosis [7-9]. According to the results from in vitro experiments, Sph-1-P exerts anti-atherosclerotic properties such as the induction of NO production [18], the suppression of TNF-?-induced VCAM-1 induction [19], and the inhibition of the adhesion of monocytes on the endothelium through the rearrangement of endothelial integrins [20] at concentrations up to several hundred nM; meanwhile, Sph-1-P can induce adhesion molecules by activating nuclear factor $\mathrm{k}-\mathrm{B}[19,21,22]$ and activate platelets $[23,24]$ at concentrations higher than several $\mu \mathrm{M}$. Therefore, Sph-1-P can create a vicious cycle formed by local thrombosis in atherosclerotic lesions; platelets are activated in the atherosclerotic lesions and Sph-1-P is released from the platelets, and then the high concentration of Sph-1-P accelerates atherosclerosis and further activates platelets. Further clinical and basic studies are needed to elucidate the association between serum Sph-1-P and atherosclerotic diseases as well as the possible existence of other unknown factors determining the release of Sph-1-P from platelets.

Concerning this issue, interesting relationships between the serum Sph-1-P and ATX levels were observed in the present study. ATX is a key enzyme in the production of LPA; LPA is hydrolyzed from LPC by ATX/ lysophospholipase D (LysoPLD) [15,16]. Along with these functions, ATX is known to hydrolyze SPC to produce Sph-1-P [17], although the contribution of this pathway to Sph-1-P might be much smaller than the 
well-known pathway in which sphingosine is converted into Sph-1-P, considering the concentration of SPC in vivo. Therefore, the observation of an inverse correlation between Sph-1-P and ATX seems contradictory. One possible mechanism is that substrates produced by ATX, such as LPA, might be involved in the formation of Sph-1-P. Further studies are needed to examine this possibility.

In this study, we measured the serum DHSph-1-P level as well as the serum Sph-1-P level. DHSph-1-P was shown to behave as an Sph-1-P receptor agonist similar to Sph-1-P [12]; however, little information is available regarding the modulation of the in vivo DHSph-1-P level. As shown in Figures 1,2 and 4, and Table 3, we demonstrated that the serum DHSph-1-P level was determined in a manner similar to the Sph-1-P level.

In summary, this study demonstrated that the serum Sph-1-P and DHSph-1-P levels were determined mainly by the platelet count and suggested that the high concentrations of Sph-1-P released from platelets might mediate cross talk between platelet activation and the formation of atherosclerotic lesions.

\section{Materials \& methods}

\section{Serum sample preparation}

Non-hematological disorder subjects [NHD] $(\mathrm{n}=33)$ and patients with aplastic anemia $[\mathrm{AA}](\mathrm{n}=2)$, essential thrombocythemia [ET] (diagnosed according to the WHO classification criteria [25] $)(\mathrm{n}=10)$, idiopathic thrombocytopenia purpura [ITP] $(\mathrm{n}=6)$, and von Willebrand diseases [VWD] $(n=3)$ were enrolled.

The serum samples used in this study were residual samples of those obtained after the completion of routine laboratory analyses. The study was approved by the Institutional Research Ethics Committee of the Faculty of Medicine, the University of Tokyo.

\section{Measurement of Sph-1-P and DHSph-1-P}

Sph-1-P and DHSph-1-P in serum samples were extracted using a two-step extraction method and were measured using high-performance liquid chromatography (HPLC) using C17-Sph-1-P as an internal standard, as previously described [26].

\section{Measurement of serum autotaxin levels}

The autotaxin (ATX) antigen levels in the serum were determined using a two-site immunoenzymetric assay with the ATX assay reagent and the TOSOH AIA system (TOSOH, Tokyo, Japan) [27]

\section{Statistical analysis}

All the data were statistically analyzed using SPSS (Chicago, IL). The results were expressed as the mean \pm SD. Values obtained from more than three groups were compared using a one-way analysis of variance (ANOVA) followed by a post-hoc test. Correlations were evaluated using the Pearson correlation. The independent effects of the RBC count, platelet count, albumin, ATX, HDL-C, LDL-C, and TG levels on the serum Sph-1-P or DHSph-1-P levels were evaluated using stepwise multiple regression analyses. P-values less than 0.05 were deemed statistically significant for all the analyses.

\section{Abbreviations}

AA: Aplastic anemia; ATX: Autotaxin; DHSph-1-P: Dihydrosphingosine 1phosphate; ET: Essential thrombocythemia; HPLC: High-performance liquid chromatography; ITP: Idiopathic thrombocytopenia purpura;

LPA: Lysophosphatidic acid; LPC: Lysophosphatidylcholine; LysoPLD: Lysophospholipase D; NHD: Non-hematological disorder; RBC: Red blood cell; Sph-1-P: Sphingosine 1-phosphate;

SPC: Sphingosylphosphorylcholine; WD: Von Willebrand disease.

\section{Competing interests}

The authors declared that they have no competing interests.

\section{Authors' contribution}

$\mathrm{YO}$ and MK participated in study design, carried out experiments and data analysis, and drafted the initial manuscript. RO participated in several experiments. $\mathrm{Kl}$ participated in measurement of serum autotaxin levels. JA, $H Y$, and MT were involved in study design and drafting manuscript. YY conceived of the study, coordinated the study design and helped to draft the manuscript. All authors read and approved the final manuscript.

\section{Acknowledgements}

This work was supported by JSPS KAKENHI Grant Number 22249017.

\section{Author details}

${ }^{1}$ Department of Clinical Laboratory, The University of Tokyo Hospital, Tokyo, Japan. ${ }^{2}$ Department of Clinical Laboratory Medicine, Graduate School of Medicine, The University of Tokyo, 7-3-1 Hongo, Bunkyo-ku, Tokyo 113-8655, Japan. ${ }^{3}$ Analytical Laboratory Chemistry, Graduate School of Health Care Sciences, Tokyo Medical and Dental University, Tokyo, Japan. ${ }^{4}$ Bioscience Division, Reagent Development Department, AIA Research Group, TOSOH Corporation, Kanagawa, Japan. ${ }^{5}$ Laboratory of Molecular and Cellular Biochemistry, Graduate School of Pharmaceutical Sciences, Tohoku University, Miyagi, Japan.

Received: 8 January 2013 Accepted: 13 February 2013 Published: 18 February 2013

\section{References}

1. Yatomi Y: Sphingosine 1-phosphate in vascular biology: possible therapeutic strategies to control vascular diseases. Curr Pharm Des 2006, 12:575-587.

2. Spiegel S, Milstien S: Sphingosine 1-phosphate, a key cell signaling molecule. J Biol Chem 2002, 277:25851-25854

3. Spiegel S, Milstien S: Sphingosine-1-phosphate: an enigmatic signalling lipid. Nat Rev Mol Cell Biol 2003, 4:397-407.

4. Yatomi Y, Igarashi Y, Yang L, Hisano N, Qi R, Asazuma N, Satoh K, Ozaki Y, Kume S: Sphingosine 1-phosphate, a bioactive sphingolipid abundantly stored in platelets, is a normal constituent of human plasma and serum. J Biochem 1997, 121:969-973.

5. Pappu R, Schwab SR, Cornelissen I, Pereira JP, Regard JB, Xu Y, Camerer E, Zheng YW, Huang Y, Cyster JG, Coughlin SR: Promotion of lymphocyte egress into blood and lymph by distinct sources of sphingosine-1 -phosphate. Science 2007, 316:295-298.

6. Ohkawa R, Nakamura K, Okubo S, Hosogaya S, Ozaki Y, Tozuka M, Osima N Yokota H, Ikeda H, Yatomi Y: Plasma sphingosine-1-phosphate measurement in healthy subjects: close correlation with red blood cell parameters. Ann Clin Biochem 2008, 45:356-363.

7. Sattler KJ, Elbasan S, Keul P, Elter-Schulz M, Bode C, Graler MH, BrockerPreuss M, Budde T, Erbel R, Heusch G, Levkau B: Sphingosine 1-phosphate 
levels in plasma and HDL are altered in coronary artery disease. Basic Res Cardiol 2010, 105:821-832.

8. Argraves KM, Sethi AA, Gazzolo PJ, Wilkerson BA, Remaley AT, TybjaergHansen A, Nordestgaard BG, Yeatts SD, Nicholas KS, Barth JL, Argraves WS: S1P, dihydro-S1P and C24:1-ceramide levels in the HDL-containing fraction of serum inversely correlate with occurrence of ischemic heart disease. Lipids Health Dis 2011, 10:70.

9. Deutschman DH, Carstens JS, Klepper RL, Smith WS, Page MT, Young TR, Gleason LA, Nakajima N, Sabbadini RA: Predicting obstructive coronary artery disease with serum sphingosine-1-phosphate. Am Heart J 2003, 146:62-68.

10. Aoki S, Yatomi Y, Ohta M, Osada M, Kazama F, Satoh K, Nakahara K, Ozaki Y: Sphingosine 1-phosphate-related metabolism in the blood vessel. J Biochem 2005, 138:47-55.

11. Kobayashi N, Nishi T, Hirata T, Kihara A, Sano T, Igarashi Y, Yamaguchi A: Sphingosine 1-phosphate is released from the cytosol of rat platelets in a carrier-mediated manner. J Lipid Res 2006, 47:614-621.

12. Van Brocklyn JR, Lee MJ, Menzeleev R, Olivera A, Edsall L, Cuvillier O, Thomas DM, Coopman PJ, Thangada S, Liu CH, et al: Dual actions of sphingosine-1-phosphate: extracellular through the Gi-coupled receptor Edg-1 and intracellular to regulate proliferation and survival. J Cell Biol 1998, 142:229-240.

13. Okajima F: Plasma lipoproteins behave as carriers of extracellular sphingosine 1-phosphate: is this an atherogenic mediator or an anti-atherogenic mediator? Biochim Biophys Acta 2002, 1582:132-137.

14. Rodriguez C, Gonzalez-Diez M, Badimon L, Martinez-Gonzalez J: Sphingosine-1-phosphate: A bioactive lipid that confers high-density lipoprotein with vasculoprotection mediated by nitric oxide and prostacyclin. Thromb Haemost 2009, 101:665-673.

15. Aoki J, Inoue A, Okudaira S: Two pathways for lysophosphatidic acid production. Biochim Biophys Acta 2008, 1781:513-518.

16. Okudaira S, Yukiura H, Aoki J: Biological roles of lysophosphatidic acid signaling through its production by autotaxin. Biochimie 2010 92:698-706.

17. Clair T, Aoki J, Koh E, Bandle RW, Nam SW, Ptaszynska MM, Mills GB, Schiffmann E, Liotta LA, Stracke ML: Autotaxin hydrolyzes sphingosylphosphorylcholine to produce the regulator of migration, sphingosine-1-phosphate. Cancer Res 2003, 63:5446-5453.

18. Igarashi J, Bernier SG, Michel T: Sphingosine 1-phosphate and activation of endothelial nitric-oxide synthase. differential regulation of Akt and MAP kinase pathways by EDG and bradykinin receptors in vascular endothelial cells. J Biol Chem 2001, 276:12420-12426.

19. Kimura T, Tomura H, Mogi C, Kuwabara A, Damirin A, Ishizuka T, Sekiguchi A, Ishiwara M, Im DS, Sato K, et al: Role of scavenger receptor class B type I and sphingosine 1-phosphate receptors in high density lipoproteininduced inhibition of adhesion molecule expression in endothelial cells. J Biol Chem 2006, 281:37457-37467.

20. Aoki S, Yatomi Y, Shimosawa T, Yamashita H, Kitayama J, Tsuno NH, Takahashi K, Ozaki Y: The suppressive effect of sphingosine 1-phosphate on monocyte-endothelium adhesion may be mediated by the rearrangement of the endothelial integrins alpha(5)beta(1) and alpha(v) beta(3). J Thromb Haemost 2007, 5:1292-1301.

21. Shimamura K, Takashiro Y, Akiyama N, Hirabayashi T, Murayama T: Expression of adhesion molecules by sphingosine 1-phosphate and histamine in endothelial cells. Eur J Pharmacol 2004, 486:141-150.

22. Kase H, Hattori Y, Jojima T, Okayasu T, Tomizawa A, Suzuki K, Banba N, Monden T, Satoh H, Akimoto K, Kasai K: Globular adiponectin induces adhesion molecule expression through the sphingosine kinase pathway in vascular endothelial cells. Life Sci 2007, 81:939-943.

23. Yatomi Y, Yamamura S, Ruan F, Igarashi Y: Sphingosine 1-phosphate induces platelet activation through an extracellular action and shares a platelet surface receptor with lysophosphatidic acid. J Biol Chem 1997 272:5291-5297.

24. Motohashi K, Shibata S, Ozaki Y, Yatomi Y, Igarashi Y: Identification of lysophospholipid receptors in human platelets: the relation of two agonists, lysophosphatidic acid and sphingosine 1-phosphate. FEBS Lett 2000, 468:189-193.

25. Swerdlow SHCE, Harris NL, Jaffe ES, Pileri SA, Stein H, Thiele J, Vardiman JW: WHO classification of tumours of haematopoietic and lymphoid tissues In Classification of tumours of haematopoietic and lymphoid tissues. 4th edition. Edited by Book WHO. Lyon, France: IARC Press; 2008:88-93.
26. Yatomi Y: Plasma sphingosine 1-phosphate metabolism and analysis. Biochim Biophys Acta 2008, 1780:606-611.

27. Nakamura K, Igarashi K, Ide K, Ohkawa R, Okubo S, Yokota H, Masuda A, Oshima N, Takeuchi T, Nangaku M, et al: Validation of an autotaxin enzyme immunoassay in human serum samples and its application to hypoalbuminemia differentiation. Clin Chim Acta 2008, 388:51-58.

doi:10.1186/1476-511X-12-20

Cite this article as: Ono et al:: Sphingosine 1-phosphate release from platelets during clot formation: close correlation between platelet count and serum sphingosine

1-phosphate concentration. Lipids in Health and Disease 2013 12:20.

\section{Submit your next manuscript to BioMed Central and take full advantage of:}

- Convenient online submission

- Thorough peer review

- No space constraints or color figure charges

- Immediate publication on acceptance

- Inclusion in PubMed, CAS, Scopus and Google Scholar

- Research which is freely available for redistribution

Submit your manuscript at www.biomedcentral.com/submit
C) Biomed Central 